\title{
Combination therapy patents: a new front in evergreening
}

\author{
As pharmaceutical companies seek patent protection for combinations of cancer therapeutics, it is worthwhile to \\ assess what constitutes an 'unexpected result' for the purpose of an appropriate patent and whether randomized, \\ controlled trials of drug combinations have the ability to generate them.
}

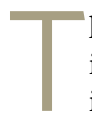
he patent system is designed so that inventors who bear the burden of innovation risks and are successful in their efforts have the potential to reap substantial rewards. From a constitutional perspective, however, the ultimate goal is not the benefit of individual inventors but the benefit to society as a whole ${ }^{1}$. The government provides particular citizens the right to exclude others from certain products or activities for a limited time, in the hope that doing so will lead to benefits for everyone ${ }^{2}$.

Pharmaceutical companies have become adept at legal and business strategies aimed at extending the period of protection, frequently through minor modifications to a drug's dosage, formulation or delivery system ${ }^{3-5}$. Commonly known as 'evergreening', these strategies allow innovators to increase the period of time during which their successful drug can generate revenue against limited competition, while imposing financial and patient care costs on society ${ }^{3,4}$. Here, we review the legal concepts of obviousness and unexpected results - terms with which both physicians and regulators (for example, the US Food and Drug Administration (FDA)) should be familiar in light of the emergence of pharmaceutical combination patents - and examine their interaction with current clinical research. In the highly profitable oncology sector, we evaluated whether pharma companies are employing a new variant of these strategies by combining 'backbone' drugs (widely accepted standards of care) with other drugs likely to be used to treat a given disease state ${ }^{3,4}$. Specifically, we focused our efforts on a subset of combination therapies developed in cancer as a case study. Using publicly available information, we assess the current patent and clinical trial landscapes for this combination. We demonstrate that this new front in evergreening not only lacks the inventive nature typically justifying the 20 -year patent reward but also leads to the design and development of clinical trials that lack even the potential to yield unexpected results characteristic of a patentable invention - a finding similar to the strategic behaviors of drug- and device-makers in generating and studying drug-device combinations ${ }^{6}$. Given the implications of these emerging strategic behaviors, we close by offering a proposal for clinical trial elements that are necessary to provide a thoughtful evaluation of the non-obviousness of combination therapy patents, as well as suggesting ways in which inter-agency collaboration at the federal level could help both industry and researchers focus on achieving optimal innovation in the public interest.

\section{A brief review of patentability}

Five canonical elements are required of an innovation for it to be patentable under US law: the invention must be of proper subject matter, useful, novel, non-obvious and the application must include proper disclosure ${ }^{7}$. Of particular relevance to combination therapy patents, the inventor must demonstrate that the invention would not have been obvious to a person having ordinary skill in the art (POSITA), which is defined as a person who has the capability to understand the scientific and engineering principles applicable to the relevant art ${ }^{8,9}$. Precise requirements vary according to the case and the invention, but in most patent cases involving drug development, the POSITA has skills commensurate with an advanced degree (for example, $\mathrm{PhD}$ or MD) along with experience in the research or treatment of the specific disease state $(s)^{10}$. As the US Supreme Court has noted, a POSITA is expected to be a person of ordinary creativity, one who is able to fit the teachings of multiple patents together like pieces of a puzzle ${ }^{8}$. Some recent courts have found that a POSITA would also be supported by the insights of a multi-disciplinary drug discovery and development team ${ }^{10}$. If an invention would be obvious to this creative POSITA who is backed by a team, then the court should reject the patent.

Importantly, the term 'invention' goes beyond the notion of tinkering.
A related concept, 'obvious to try', holds that an invention is unpatentable when it is comprised of a set of re-combined elements, the various permutations of which would be predictable to try ${ }^{8}$. Absolute predictability is not required, nor does it matter whether the trials of the re-combined elements require extensive time, money and effort to test ${ }^{11}$. Rather, the invention is unpatentable if there is a finite number of identified solutions - that is, a set of things to try - and a POSITA would have a reasonable expectation of success if he or she were to try each of these solutions in turn ${ }^{8,11}$. Multiple courts have explicitly stated that it is normal for scientists to optimize each of the variables in a known process to improve on what is generally known already - but that acts of optimization are not themselves patentable $\mathrm{e}^{11,12}$.

A claimant can potentially overcome obviousness rejections by demonstrating what are known as objective indicia of an invention's non-obviousness. These may include achievement of commercial success, fulfillment of a long felt but unsolved need, prior unsuccessful attempts by other POSITAs to solve the problem, evidence that the field's conventional wisdom 'teaches away' from the inventor's solution, or unexpected results that a POSITA would not anticipate ${ }^{8}$. Among the objective indicia, generation of unexpected results is the most common avenue for successfully demonstrating non-obviousness, particularly for pharmaceutical inventions $s^{13,14}$. As courts explain, the principle behind the doctrine of unexpected results is straightforward: an invention that exhibits a superior characteristic or advantage in a way that surprises even a skilled artisan immersed in the field is clearly not obvious ${ }^{15}$. Courts have determined that for a result to be unexpected, it must differ from the POSITA's expected results in kind rather than degree ${ }^{16}$. In the context of a combination of two drugs that are each effective when given sequentially, the patent claimant must demonstrate that 


\section{Box 1 | Key principles of oncology clinical trial design}

Rather than hard and fast 'laws', the following generally accepted precepts in oncology clinical trials and oncology clinical practice are known to both practicing and researching oncologists alike $^{23}$. Consequently, these precepts help to inform oncology's prior art.

\section{Precept 1: combination and} progression-free survival Begin with two hypothetical drugs, A and $B$, with different mechanisms of action, each of which independently increases the amount of time that a tumor does not grow, or progression-free survival (PFS), in a disease state. Combining $\mathrm{A}$ and $\mathrm{B}$ would be expected to extend PFS (as well as, possibly, the amount of time that the patient lives, called overall survival (OS), more than either of the individual drugs alone.

If $\mathrm{PFS}_{\mathrm{A}}>0$ and $\mathrm{PFS}_{\mathrm{B}}>0$, and $\mathrm{OS}_{\mathrm{A}}>0$ and $\mathrm{OS}_{\mathrm{B}}>0$, then none of the following are unexpected results:

$$
\begin{gathered}
\mathrm{PFS}_{\mathrm{A}+\mathrm{B}}>\mathrm{PFS}_{\mathrm{A}} \\
\mathrm{PFS}_{\mathrm{A}+\mathrm{B}}>\mathrm{PFS}_{\mathrm{B}} \\
\mathrm{OS}_{\mathrm{A}+\mathrm{B}}>\mathrm{OS}_{\mathrm{A}} \\
\mathrm{OS}_{\mathrm{A}+\mathrm{B}}>\mathrm{OS}_{\mathrm{B}}
\end{gathered}
$$

\section{Precept 2: sequential administration and PFS}

Sequentially administering individually active chemotherapy agents is a standard approach taken by practicing oncologists in treating an incurable malignancy. The goal is to extract maximal benefit from a single drug before the tumor achieves resistance to that drug. Administering up front and in combination two drugs that may otherwise be administered sequentially may increase PFS without impacting OS.

the properties of the combination are specifically the result of having combined the drugs, rather than reflecting properties of one or both of the drugs, given alone or sequentially. Consider, for example, two modestly effective drugs that each improves survival by two months when given in sequence. Prolongation of survival by, say, not two months but two years
If $\mathrm{PFS}_{\mathrm{A}}>0$ and $\mathrm{PFS}_{\mathrm{B}}>0$, then neither of the following are unexpected results:

$$
\begin{gathered}
\mathrm{PFS}_{\mathrm{A}+\mathrm{B}}>\mathrm{PFS}_{\mathrm{A} \rightarrow \mathrm{B}} \\
\mathrm{PFS}_{\mathrm{A}+\mathrm{B}}>\mathrm{PFS}_{\mathrm{B} \rightarrow \mathrm{A}}
\end{gathered}
$$

\section{Precept 3: structurally similar drugs} behave similarly

When used in the same disease state, drugs with the same mechanism of action are likely to confer similar PFS and OS benefit.

If $\mathrm{A} \cong \mathrm{C}$ or $\mathrm{B} \cong \mathrm{D}, \mathrm{PFS}_{\mathrm{A}}>0$ and $\mathrm{PFS}_{\mathrm{B}}>$ 0 , and $\mathrm{OS}_{\mathrm{A}}>0$ and $\mathrm{OS}_{\mathrm{B}}>0$, then none of the following are unexpected results:

$$
\begin{gathered}
\mathrm{PFS}_{\mathrm{A}+\mathrm{B}}>\mathrm{PFS}_{\mathrm{C}} \\
\mathrm{PFS}_{\mathrm{A}+\mathrm{B}}>\mathrm{PFS}_{\mathrm{D}} \\
\mathrm{OS}_{\mathrm{A}+\mathrm{B}}>\mathrm{OS}_{\mathrm{C}} \\
\mathrm{OS}_{\mathrm{A}+\mathrm{B}}>\mathrm{OS}_{\mathrm{D}}
\end{gathered}
$$

\section{Precept 4: the straw man}

Many drugs, administered as single agents with palliative intent in later lines of therapy, appear to prolong PFS and OS by clinically relevant amounts of time, but only when compared to the counterfactuals of administering a poorly performing drug or no therapy at all. Combining two active drugs with different mechanisms of action will likely confer statistically and clinically significant PFS and OS benefit when compared to the third drug.

If $\mathrm{PFS}_{\mathrm{A}}>0$ and $\mathrm{PFS}_{\mathrm{B}}>0, \mathrm{OS}_{\mathrm{A}}>0$ and $\mathrm{OS}_{\mathrm{B}}>0$, and both $\mathrm{PFS}_{\mathrm{E}}$ and $\mathrm{OS}_{\mathrm{E}} \rightarrow 0$, then neither of the following are unexpected results:

$$
\begin{gathered}
\mathrm{PFS}_{\mathrm{A}+\mathrm{B}}>\mathrm{PFS}_{\mathrm{E}} \\
\mathrm{PFS}_{\mathrm{A}+\mathrm{B}}>\mathrm{OS}_{\mathrm{E}}
\end{gathered}
$$

and an observed decrease in side effects by administering the two drugs as a combination would be an unexpected result.

\section{Combination therapy in medicine}

The prior art. Combining two active therapies into a single regimen is a common practice in medicine: angiotensin-converting enzyme inhibitors and diuretics in hypertension, long-acting beta agonists and inhaled corticosteroids in chronic obstructive pulmonary disease, and combinations of antiretrovirals and protease inhibitors in HIV are but three widely prescribed examples. Oncology is particularly fertile ground, as chemotherapy combinations have been used to treat cancer for well over a half-century, leading to the cures of select malignancies in the 1960s and $1970 \mathrm{~s}^{17-21}$. Consequently, combining two or more drugs has become a standard approach in the treatment of the vast majority of cancers $^{22}$. Testing whether patients who receive the combination of therapies $A$ and $\mathrm{B}(\mathrm{A}+\mathrm{B})$ have improved outcomes, relative to those who receive either $\mathrm{A}$ or $\mathrm{B}$ alone, became a standard approach in oncology drug development ${ }^{23}$

With decades of accumulated experience, key principles of oncology clinical trial design have been derived and are distilled in Box 1. These precepts are understood not only by the sophisticated multi-disciplinary drug discovery and development teams of the modern age but, at the very least, are intuited by clinical practitioners. Combining active anticancer drugs in the search for more efficacious regimens has been employed for decades. Consequently, the resulting patent claims may be entirely unpatentable, as the combination approach has long existed in the prior art and was obvious to try. In light of these obviousness concerns, the question becomes whether a clinical trial - in any subspecialty of medicine - might produce results so surprising that they would meet the threshold of 'unexpected'.

Discovering the unexpected. From the clinical trial precepts in Box 1 flow features necessary of a clinical trial of an oncology combination therapy $(\mathrm{A}+\mathrm{B})$ to demonstrate an unexpected result. First, clinical trial participants ought to be randomly assigned to either the intervention or the comparator $\operatorname{arm}(\mathrm{s})$ - they must be randomized controlled trials (RCTs). Randomization helps ensure that the observed outcome of the clinical trial is due to differences in the treatments rather than biased treatment arm assignment. Without randomization, differences in outcomes can be driven by baseline patient characteristics, rather than differences in the treatments.

Second, the most appropriate comparator $\operatorname{arm}(\mathrm{s})$ is a sequence (or sequences) of the two drugs that make up the combination (for example, $\mathrm{A} \rightarrow \mathrm{B}$ and/or $\mathrm{B} \rightarrow \mathrm{A}$ ). If $\mathrm{A}+$ $\mathrm{B}$ confers 12 months of survival while $\mathrm{A} \rightarrow \mathrm{B}$ confers 6 months of survival, then combining $A$ and B is clearly advantageous. Alternatively, if the comparator arm is, say, A followed by something else, then claims about the benefit 


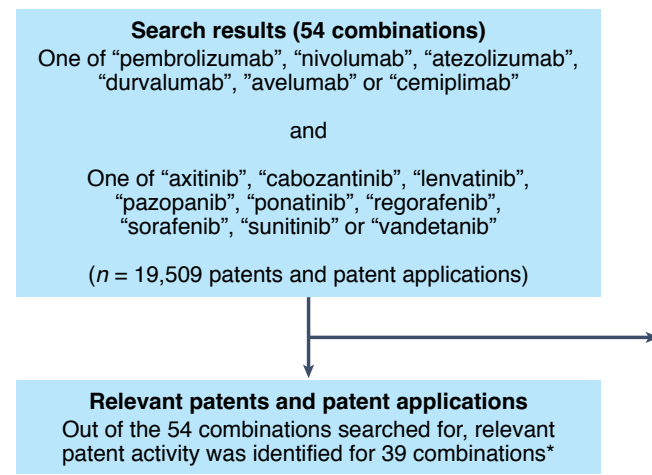

Excluded patents and patent applications that: - Were not specifically drafted for one or more of the searched combinations

- Were not filed by the owner company of the kinase inhibitor or monoclonal antibody in question

question

Required additional components beyond the combination therapy, such as other antibodies or proteins

Fig. 1 | Patent search strategy. TotalPatentOne (LexisNexis), a repository of patents and patent applications, was searched for patents and patent applications covering the combinations of VEGF-inhibiting small molecules and IO drugs listed. *Note: for each of the 15 combinations where initial analysis did not yield any patents or patent applications meeting our criteria for inclusion, it remains possible that, within the thousands of claims in these patents or others, there exist ones that could be asserted for the purpose of evergreening the combination therapy.

act of combining cannot be made. Clinical trials that allow crossover from comparator to intervention arm at progression are unable to make strong conclusions about overall survival (OS - the amount of time between randomization and the patient's death) without assuming that subsequent treatments are similar in their effects on outcomes ${ }^{24}$.

Only utilization of pre-determined sequences of therapy allow for meaningful comparison, albeit with limitation of freedom of choice at first progression.

Finally, the primary outcome measure of the RCT ought to be OS. This is contrasted with progression-free survival (PFS), a surrogate outcome that measures the time between randomization and the patient's disease getting worse (progressing) that has controversially been relied on for recent regulatory decisions ${ }^{25,26}$. As demonstrated in Box 1, due to the nature of combining individually active therapies, none of the possible patentable PFS results would be unexpected: given the individual activity in the disease state of both $A$ and $B$, the POSITA would expect $A+B$ to have equal or greater PFS benefit to $\mathrm{A} \rightarrow \mathrm{B}$ or $\mathrm{B} \rightarrow \mathrm{A}$, and if $A+B$ has lower PFS benefit, then the point is moot.

Armed with a basic understanding of patentability, oncology's prior art, and principles of clinical trials that ought to be present to enable identification of truly unexpected results, we can now turn our attention to real-world applications. Let us examine an emerging combination therapy in oncology as a case example.

\section{Evergreening combinations}

The results of analyses in numerous clinical trials suggesting the exceptional benefit of combining vascular endothelial growth factor (VEGF) inhibitors with immune checkpoint blockade (also referred to as immunotherapy (IO)) were presented at the European Society of Molecular Oncology 2020 Annual Meeting ${ }^{27-30}$. VEGF and IO have individually demonstrated efficacy in a variety of cancers, including advanced and metastatic renal cell carcinoma (mRCC) ${ }^{31,32}$. VEGF inhibitors reduce a tumor's growth by blocking its ability to recruit and build a blood supply, while IO acts by inhibiting the signals that a cancer uses to evade the body's immune system ${ }^{33}$. VEGF inhibitors may also increase intratumoral T-cell infiltration, an immunologic change that likely enhances IO's anticancer efficacy ${ }^{34}$.

Given the decades-long approach in oncology of combining drugs with different mechanisms of action, it would be obvious for a POSITA to consider combining these two therapies. In fact, VEGF-IO combinations have been hypothesized since 2006 (ref. ${ }^{34}$ ), trialed in a variety of cancers ${ }^{33}$, and have since become, arguably, standard of care for the first-line treatment of advanced or $\mathrm{mRCC}^{35}$. All of this occurred before the 2020 trial result announcements.

The VEGF-IO patent landscape. Given the ongoing clinical investigation surrounding VEGF-IO, it would be consistent with the self-interested behavior of a corporation to seek out patent protection for this combination. We therefore set out to determine whether substantial patent activity - evidence beyond anecdote is occurring for combinations of drugs ordinarily known by relevant oncology practitioners. Given oncology's prior knowledge, these combinations are likely to constitute obvious (or obvious to try) formulations ${ }^{8}$. We searched TotalPatent One (LexisNexis) during July and August 2020 for 54 combinations of drugs, containing one each of FDA-approved VEGF or multi-tyrosine kinase inhibitors - axitinib, cabozantinib, lenvatinib, pazopanib, ponatinib, regorafenib, sorafenib, sunitinib, and vandetanib and FDA-approved programmed cell death protein 1 (PD-1)-axis-inhibiting IO monoclonal antibodies - pembrolizumab, nivolumab, atezolizumab, durvalumab, avelumab and cemiplimab.

Our database search yielded 19,509 patents and patent applications (Fig. 1). Among these, we considered only patents filed by the owner company of the VEGF drug or IO monoclonal antibody in question, given that such patents are the most likely to suggest that a company is attempting to evergreen the protections on an existing drug therapy ${ }^{3}$. To verify that at least some of the claims within the patents were specifically drafted to the drug combination in question, and to eliminate any patents that specified additional components beyond the combination therapy, such as other antibodies or proteins, we examined in detail those patents determined to be the most relevant for a given drug combination. Selection of the most relevant patent is best illustrated with an example: if two similar patent applications were identified for the same drug combination, one mentioning the drug combination in the specifications and the other mentioning it in both the specifications and the claims, we evaluated the latter. Once an example of patent activity meeting these criteria for one of the 54 drug combinations was identified, we proceeded to the subsequent drug combination.

In this manner, we identified patent activity pertaining to 39 of the 54 drug combinations searched, presented in Supplementary Table 1. The results demonstrate that, across a variety of specific formulations, those companies likely to have incentive to engage in evergreening behavior have been, and continue to be, engaged in substantial patent activity for drug combinations known to those in the field. Given the discussion above and the potential implications of evergreening, this area of patenting and drug development merited critical evaluation.

Teaching away. A patent claimant can overcome obviousness concerns by demonstrating objective indicia of non-obviousness, such as when the prior art teaches away from an invention. That is, 


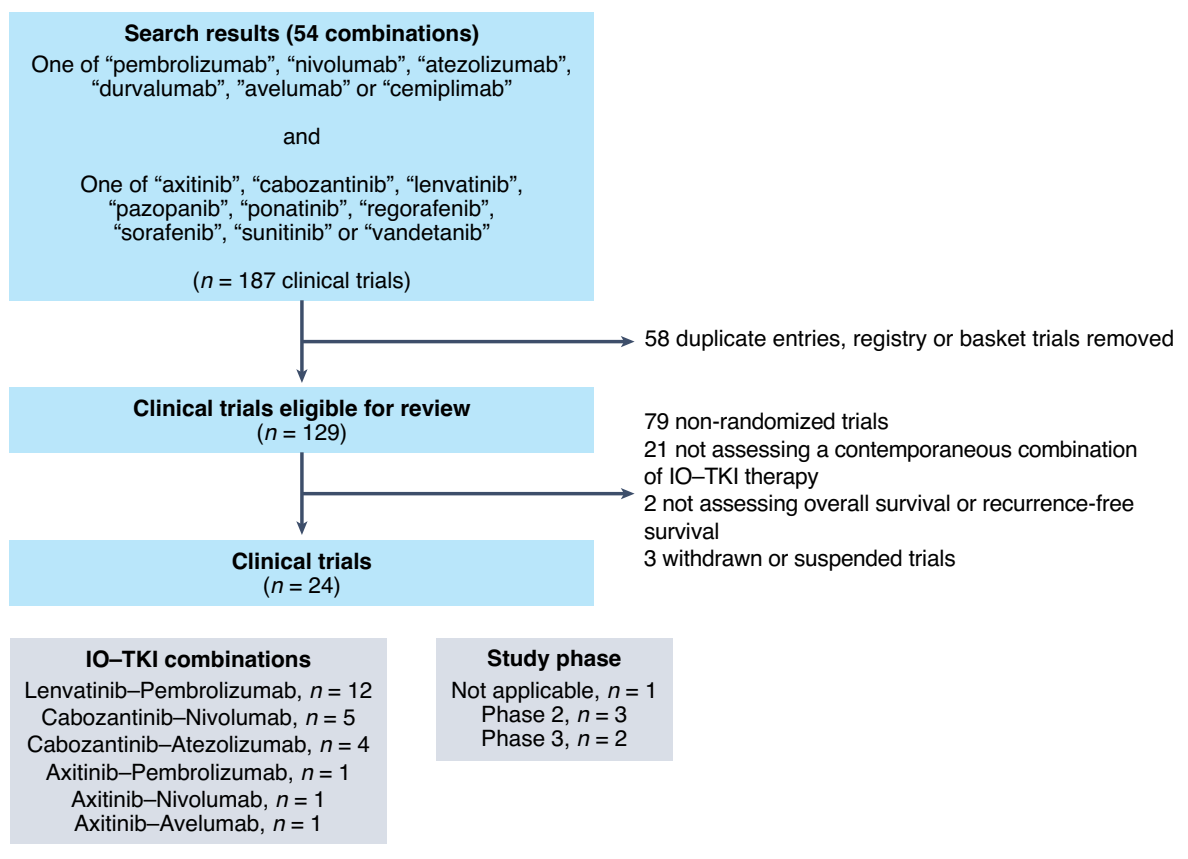

Fig. 2 | Identification of clinical trials evaluating the combination of VEGF inhibitors and IO agents from ClinicalTrials.gov. Starting with search results of 187 clinical trials, 129 distinct clinical trials were identified. Out of these, 105 trials were excluded from further analysis for non-randomized design, failure to utilize contemporaneous combination or withdrawal/suspension of the study. The remaining 24 RCTs were included in the final analysis. TKI, tyrosine kinase inhibitor; IO, immunotherapy.

was there evidence in the prior art telling inventors not to combine VEGF and IO? Early phase 1 clinical trial work conducted between 2012 and 2014 and publicly disclosed in 2014 evaluated two VEGF-IO combinations in mRCC. In these clinical trials, sunitinib-nivolumab and pazopanibnivolumab demonstrated approximately $70 \%$ and $60 \%$, respectively, grade $3 / 4$ adverse event rates ${ }^{36,37}$. A subsequent phase $1 / 2$ study of pazopanib-pembrolizumab in $\mathrm{mRCC}$ conducted between 2013 and 2017 and publicly disclosed in 2017 demonstrated grade $3 / 4$ adverse events of $>80 \%$ and at the time concluded that certain VEGF-IO combinations were unsuitable for RCTs ${ }^{38,39}$. At first glance, these studies - having raised the possibility of a VEGF-IO combination conferring prohibitive toxicity - may have taught away from VEGF-IO combination therapy. To the POSITA with experience in drug discovery and development, though, the observed toxicities were 'off-target' effects of the particular VEGF drugs tested that would not be expected to generalize to all VEGF inhibitors, as demonstrated by the first-in-class axitinib-pembrolizumab combination $^{40}$.

VEGF-IO clinical trials. As the most commonly used objective indicium for overcoming obviousness concerns is demonstration of an unexpected result, we asked whether the RCTs in the VEGF-IO development space have the capacity to demonstrate an unexpected result, if one were to exist. To examine this landscape, we searched the National Clinical Trials Registry (ClinicalTrials.gov) on 20 August 2020 for each of the 54 combinations of one of the above VEGF inhibitors and one of the above IO drugs. Building on two of the key clinical trial principles described above (1) randomization; and (2) evaluation of OS as a primary or co-primary endpoint - we excluded any clinical trials that were not randomized; did not contemporaneously administer one of the above combinations; assessed a primary endpoint(s) other than OS, PFS or recurrence-free survival; or indexed in ClinicalTrials.gov as having a status other than 'active, not recruiting' or 'completed' (Fig. 2).

We identified 24 eligible RCTs, summarized in Supplementary Table 2, with total anticipated enrollment of 14,614 patient volunteers. Twelve (50\%) trials evaluate lenvatinib-pembrolizumab and cabozantinib-nivolumab and cabozantinibatezolizumab are each evaluated by four (17\%). Twenty-one (88\%) are phase 3 trials, and the remaining three (13\%) are randomized phase 2 trials. Nineteen (79\%) are industry-funded trials and five $(21 \%)$ are government-funded. These trials used five dominant designs:

Type 1: A + B versus A;

Type 2: A + B versus B;

Type 3: A + B versus C;

Type 4: $A+B$ versus $D$;

Type 5: A + B versus E;

in which $A$ is one of the VEGF inhibitors listed above, $\mathrm{B}$ is one of the IO agents listed above, $\mathrm{C}$ is an established and related VEGF inhibitor (for example, sunitinib), D is an established but unrelated standard of care (for example, chemotherapy, physician's choice) and $\mathrm{E}$ is a non-active comparator (placebo or otherwise).

Importantly, none of the 24 eligible RCTs possess the three criteria needed to demonstrate an unexpected result that were derived above: randomization, sequential treatment with $\mathrm{A}$ and $\mathrm{B}$ as a comparator, and OS as the primary or co-primary endpoint. The absence of sequential treatments is clear. Thirteen of the 24 eligible RCTs employ comparator arms that include at least one drug from the VEGF-IO combination. None incorporate $\mathrm{A} \rightarrow \mathrm{B}$ or $\mathrm{B} \rightarrow \mathrm{A}$ as a comparator, and there is no pre-specified OS or PFS analysis of $\mathrm{A}+\mathrm{B}$ compared to $\mathrm{A} \rightarrow$ $\mathrm{B}$ or $\mathrm{B} \rightarrow \mathrm{A}$ subpopulations. Consequently, these RCTs cannot demonstrate an unexpected result attributable to the act of combining ${ }^{6}$. Nine of the 13 trials that include either A or B as a component of the comparator arm employ OS as the primary or co-primary endpoint (Supplementary Table 2). Representative RCTs are discussed in Box 2.

Importantly, five (21\%) type-3 trials were identified. Four of these trials were conducted in mRCC and compared VEGF-IO combinations to sunitinib; all have reported results. First, axitinibavelumab, in the JAVELIN Renal-101 study reported on 21 March 2019, failed to demonstrate benefit of the combination ${ }^{41}$. Second, axitinib-pembrolizumab, in the KEYNOTE-426 study also reported on 21 March 2019, demonstrated an OS benefit ${ }^{35}$. The remaining two RCTs, evaluating cabozantinib-nivolumab and lenvatinibpembrolizumab, continued to randomize patients to sunitinib control arms after the announcement of KEYNOTE-426 results ${ }^{42,43}$. Nearly 24 months after KEYNOTE-426 publication, in March 2021, the CheckMate 9ER trial of cabozantinib-nivolumab was published, demonstrating modest PFS and OS benefits ${ }^{44}$; FDA approval had occurred 6 weeks earlier ${ }^{45}$. One month later, in April 2021, the CLEAR trial evaluating lenvatinibpembrolizumab in mRCC failed to demonstrate any more than a modest benefit in $\mathrm{OS}^{46}$, yet FDA approval followed 4 months later $^{47}$. Complicating interpretation of the 


\section{Box 2 | Representative randomized controlled trials}

\section{The most common combination}

Twelve of the eligible RCTs evaluate the lenvatinib-pembrolizumab combination, but as presently designed none have the design features needed to demonstrate an unexpected result ${ }^{51-62}$. Seven of the lenvatinib-pembrolizumab RCTs incorporate pembrolizumab or lenvatinib monotherapy as a comparator arm ${ }^{51-57}$. Only two of the lenvatinib-pembrolizumab RCTs fail to utilize OS as a co-primary endpoint ${ }^{58,59}$. Critically, none of the lenvatinib-pembrolizumab trials require crossover to lenvatinib (in the case of pembrolizumab) or pembrolizumab (in the case of lenvatinib) at the time of disease progression.

None of the trials utilize OS as the sole primary endpoint. After having learned that the FDA will award approvals to VEGF-IO combination therapies on the basis of PFS benefit ${ }^{63}$, trial sponsors lack the incentive to wait for an OS endpoint to read out before seeking regulatory approval. All but one of the clinical trials involving the combination of lenvatinib and pembrolizumab are industry-sponsored and expect to enroll 7,038 patients; the one trial sponsored by a public entity will enroll 192 patients $^{59}$.

\section{How trials can be skewed}

The LEAP-008 RCT evaluates lenvatinibpembrolizumab against docetaxel or lenvatinib monotherapy as second-line treatment for metastatic non-small cell lung cancer that had progressed despite prior PD-1-axis-inhibiting therapy. It serves as an example for the myriad ways in which clinical trial design can be 'gamed' to achieve a desired outcome. First, despite the publication of evidence demonstrating modest OS benefit with the addition of ramucirumab to docetaxel four years before its opening ${ }^{64}$, LEAP-008 fails

mRCC VEGF-IO trials still further, nearly all enrolled a substantial number of patients from countries where access to IO only occurs through clinical trials, increasing the likelihood that patients randomized to the sunitinib arms of these studies would never receive standard-of-care, second-line IO in the event of disease progression, and thereby overestimating the OS benefit of VEGF-IO ${ }^{48}$.

\section{Recommendations}

Reviewing the available evidence, the RCTs of VEGF-IO combinations appear designed to incorporate VEGF-inhibiting therapy in the comparator arm. Second, although lenvatinib is one of the comparator arms of the study, no crossover to pembrolizumab monotherapy after progression on lenvatinib is mandated. Third, given the long half-life and target engagement of $\mathrm{IO}^{65}$, it is possible that lenvatinib administered in short succession following progression through pembrolizumab may 'rescue' durable IO response. LEAP-008, however, incorporates a 4-week washout period, reducing the probability of observing this phenomenon in a sequential administration paradigm. Fourth, LEAP008 employs an unusual randomization schema of 4:4:1 to lenvatinibpembrolizumab to docetaxel to lenvatinib. Despite stratification on whether IO was a remote therapy or the immediate prior therapy, LEAP-008's ability to disentangle lenvatinib's relative contribution to the efficacy of lenvatinib-pembrolizumab is likely to be limited due to only 45 patients receiving lenvatinib monotherapy. Due to these issues, LEAP-008 cannot demonstrate an unexpected result.

\section{How do government-funded RCTs do?} Lest we think these design flaws only occur in industry-sponsored clinical trials, NCT03595124 is a National Cancer Institute-funded RCT opened in July 2018 comparing axitinib-nivolumab $(\mathrm{A}+\mathrm{B})$ to axitinib $(\mathrm{A})$ and nivolumab (B) monotherapy arms in transcription factor E family translocation-positive $\mathrm{RCC}^{66}$. Crossover in the comparator arms at the time of progression from axitinib to nivolumab $(\mathrm{A} \rightarrow \mathrm{B})$ or nivolumab to axitinib $(B \rightarrow A)$ is not pre-specified, however, and the study's primary endpoint is PFS. Despite reasonable first choices for comparator arms, NCT03595124 cannot demonstrate an unexpected result.

to achieve FDA approval, rather than identify truly unexpected or scientifically novel results that would overcome obviousness concerns. While such approvals will yield significant financial rewards for the drug sponsor(s), they should not result in extension of exclusivity in the absence of a demonstration of non-obviousness through the discovery of unexpected results.

In truth, encouraging combination patents and RCTs that do not aim to identify unexpected results carries societal costs. First, the limited resources that can be dedicated to research and development efforts are directed away from the truly innovative approaches that represent the constitutional goal of the patent system and toward the commercialization of combinations that are, in the context of oncology's prior art, non-innovative. Second, even if VEGF-IO patents were to be invalidated in court, the time and expense required to challenge them often deters competitors from entering the market, interfering with the natural competitive forces expected to discipline high prices ${ }^{4}$. In short, this new frontier in evergreening raises serious societal concerns and requires coordinated action from all involved parties - from the end users who have demand for these products and the government-supported entities that enable patent-seeking research rather than true innovation, through to the executive branch entities responsible for conferring patents.

Physicians, as end users of combination therapies, bear a societal responsibility to be thoughtful producers and consumers of biomedical research - industry-sponsored or not. As with any therapy, physicians should reflect on the designs of the relevant combination therapy clinical trials. In the context of cancer, oncologists need to disabuse themselves of the notion that 'more therapy is better and in the best interests of the patient'. Learning health systems would be wise to assess the real-world outcomes of patients treated with combination therapies, especially as the gap between performance as observed in clinical trials versus real life yawns. Finally, disclosure of the shortcomings of clinical trial design is an absolutely necessary component in the desired goal of clinical care - shared clinical decision-making.

Clinical trialists and institutions have critical roles in the current pharmaceutical research and development landscape; it is this infrastructure that enables both innovative and non-innovative research. Realizing that the potential benefits of a clinical trial redound to patients other than the ones for which they care, clinical trialists must ask themselves, "Is the trial on which I am proposing to enroll patients something new or is it an incremental step?" It is unlikely physicians can do this alone, but demanding more from the research that we and others design and enact is a good first step, along with thoughtful skepticism toward certain types of patent claims. Similarly, the academic research infrastructure, as well as the cachet, on which many non-innovative clinical trials rest is supported in no small part by federal grants. Within the context of oncology, the National Cancer Institute designated 53 Comprehensive Cancer Centers in the 
United States, with federal grant support (P30) exceeding US\$265 million in fiscal year 2020 (ref. ${ }^{49}$ ). Incorporating review of the relative amounts of industry- versus non-industry-sponsored research activity occurring at a given cancer center in the NCI P30 grant renewal process may help to promote truly innovative research. The concept of taxpayers 'paying twice' for drug development - once through government-funded research and again in the form of high drug prices - has gained traction during the COVID-19 pandemic and should be seriously considered.

As the guardian of the patent and drug-making processes, coordinated federal action on the part of the US Patent and Trademark Office (USPTO) and FDA is important. As the prior arts of medicine's subspecialties become increasingly complex, the case for inter-agency collaboration between the USPTO and FDA becomes stronger. The FDA has the capacity to provide information about all registered clinical trials, fully informing the prior art and context that the USPTO requires to make its determinations. The FDA also has a supply of experts who can provide additional perspective for USPTO examiners, who are rarely (if ever) clinicians (for example, physicians, pharmacists) or pharmaceutical scientists - FDA experts would be, to a first approximation, the government's best proxy for a well-informed POSITA. In addition, the USPTO is optimally positioned to communicate to researchers and industry the requirements for patentability and to issue guidance on what constitutes unexpected results (positive or negative) in clinical trials.

The challenge in designing interagency cooperation is to ensure that the process is meaningful, rather than pro forma or simply burdensome. Fortunately, the USPTO has a model in place to better inform prior art determinations. In 2019, USPTO director Andrei Iancu reported to the Senate Judiciary Subcommittee on Intellectual Property that the USPTO was piloting projects to help examiners better identify prior art by collaboration between multiple USTPO examiners, as well as examiners from foreign patent offices ${ }^{50}$. This project could be expanded to include expertise closer to home - specifically at the FDA. Such guidance for the USPTO is in the interests of both drug developers (by providing a measure of certainty) and the public (by enhancing the risk-benefit calculus of biomedical research). Similarly, any regulatory standards that emerge at the USPTO could be incorporated by the FDA into its oversight of clinical trials and more fully inform its safety and efficacy determinations. This would allow both agencies to better carry out their mandates in serving the public interest.

\section{Garth W. Strohbehn (D) 1,2,7, , \\ Alec J. Kacew', Daniel A. Goldstein 2,3,4,5, Robin C. Feldman (D) $6,8 \bowtie$ and \\ Mark J. Ratain ${ }^{1,2} \otimes$}

${ }^{1}$ Section of Hematology/Oncology, Department of Medicine, University of Chicago, Chicago, IL, USA. ${ }^{2}$ Optimal Cancer Care Alliance, Ann Arbor, MI, USA. ${ }^{3}$ Tel Aviv University, Tel Aviv, Israel. ${ }^{4}$ Davidoff Cancer Center, Rabin Medical Center, Petah Tikva, Israel. ${ }^{5}$ Department of Health Policy and Management, Gillings School of Public Health, University of North Carolina, Chapel Hill, NC, USA. ${ }^{6}$ Center for Innovation, University of California Hastings College of the Law, San Francisco, CA, USA. ${ }^{7}$ Present address: Veterans Affairs Center for Clinical Management and Research, Ann Arbor, MI, USA. ${ }^{8}$ These authors contributed equally: Garth W. Strohbehn, Robin Feldman.

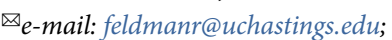

mratain@medicine.bsd.uchicago.edu

Published online: 8 December 2021

https://doi.org/10.1038/s41587-021-01137-6

\section{References}

1. Woodbridge v. U.S. 26350 (Supreme Court, 1923).

2. Feldman, R. Colum. Sci. Tech. L. Rev. 17, 30-89 (2016).

3. Feldman, R. J. Law Biosci. 5, 590-647 (2018).

4. Gaudry, K. S. Nat. Biotechnol. 29, 876-878 (2011).

5. Gowda, V., Beall, R. F., Kesselheim, A. S. \& Sarpatwari, A. Nat. Biotechnol. 39, 414-417 (2021).

6. Beall, R. F. \& Kesselheim, A. S. Nat. Biotechnol. 36, 142-145 (2018).

7. Conditions for Patentability; Non-Obvious Subject Matter 35 USC $\$ 103$ (US Congress, 2011).

8. KSR Int'l Co. v. Teleflex Inc. 550 US 398 (Justia, 2007).

9. Manual of Patent Examining Procedure (MPEP) Sec. 2141.03 (USPTO, 2020).

10. Novartis Pharms. Corp. v. Accord Healthcare, Inc. (Lexis, 2020).

11. Pfizer v. Apotex. 4801348 F.3d (Federal Circuit, 2007).

12. In re: Peterson. 3151325 F.3d (Federal Circuit, 2003).

13. Thomas, N. A. NYU L. Rev. 86, 2070-2112 (2011).

14. Pitlick, H. A. J. Pat. Trademark Off. Soc. 86, 169-182 (2004).

15. In re: Soni. 54746 F. 3d (Federal Circuit, 1995).

16. Galderma Labs., LP. v. Tolmar, Incl. 737731 F.3d (Federal Circuit, 2013).

17. DeVita, V. T. Jr. Br. J. Haematol. 122, 718-727 (2003).

18. Frei, E. III et al. Blood 13, 1126-1148 (1958)

19. Frei, E. III Cancer 18, 1580-1584 (1965).

20. Einhorn, E. H. Clin. Cancer Res. 3, 2630-2632 (1997).

21. Bagley, C. M. Jr, Young, R. C., Canellos, G. P. \& DeVita, V. T. N. Engl. J. Med. 287, 856-862 (1972).

22. National Comprehensive Cancer Network. https://www.nccn.org/ guidelines/category_1 (2020).

23. Gyawali, B. \& Prasad, V. Nat. Rev. Clin. Oncol. 14, 521-522 (2017). 24. Prasad, V. \& Grady, C. Contemp. Clin. Trials 37, 167-169 (2014). 25. Kim, C. \& Prasad, V. JAMA Intern. Med. 175, 1992-1994 (2015). 26. Kovic, B. et al. JAMA Intern. Med. 178, 1586-1596 (2018).

27. Choueri, T. K. et al. Ann. Oncol. https://oncologypro.esmo.org/ meeting-resources/esmo-virtual-congress-2020/nivolumabcabozantinib-vs-sunitinib-in-first-line-treatment-foradvanced-renal-cell-carcinoma-first-results-from-therandomized-phase-iii-checkmate-9er-trial (2020).

28. Arance Fernandez, A. M. et al. Ann. Oncol. https://oncologypro. esmo.org/meeting-resources/esmo-virtual-congress-2020/ lenvatinib-len-plus-pembrolizumab-pembro-for-advancedmelanoma-mel-that-progressed-on-a-pd-1-or-pd-11-inhibitorinitial-results-of-leap-004 (2020).

29. Lwin, Z. et al. Ann. Oncol. https://oncologypro.esmo.org/ meeting-resources/esmo-virtual-congress-2020/leap-005-phaseii-study-of-lenvatinib-len-plus-pembrolizumab-pembro-in-pati ents-pts-with-previously-treated-advanced-solid-tumours (2020).
30. Pal, S. Ann. Oncol. https://oncologypro.esmo.org/meetingresources/esmo-virtual-congress-2020/cabozantinib-c-incombination-with-atezolizumab-a-as-first-line-therapy-foradvanced-clear-cell-renal-cell-carcinoma-ccrcc-resultsfrom-the (2020).

31. Motzer, R. J. et al. J. Clin. Oncol. 27, 3584-3590 (2009).

32. Sternberg, C. N. et al. J. Clin. Oncol. 28, 1061-1068 (2010).

33. Ciciola, P., Cascetta, P., Bianco, C., Formisano, L. \& Bianco, R J. Clin. Med. 9, 675 (2020).

34. Dirkx, A. E. et al. FASEB J. 20, 621-630 (2006).

35. Rini, B. I. et al. N. Engl. J. Med. 380, 1116-1127 (2019).

36. Amin, A. et al. Am. Soc. Clin. Oncol. https://doi.org/10.1200/ jco.2014.32.15_suppl.5010 (2014).

37. Amin, A. et al. J. Immunother. Cancer 6, 109 (2019).

38. Chowdhury, S. et al. Am. Soc. Clin. Oncol. https://doi.org/ 10.1200/JCO.2017.35.15_suppl.4506 (2017).

39. Chowdhury, S. et al. Clin. Genitourin. Cancer 19, 434-446 (2021)

40. Atkins, M. B. et al. Lancet Oncol. 19, 405-415 (2018).

41. Motzer, R. J. et al. N. Engl. J. Med. 380, 1103-1115 (2019).

42. ClinicalTrials.gov (National Library of Medicine, accessed 1 November 2021); https://clinicaltrials.gov/ct2/history/NCT03141 $177 ? \mathrm{~A}=38 \& \mathrm{~B}=39 \& \mathrm{C}=$ Side-by-Side\#StudyPageTop

43. ClinicalTrials.gov (National Library of Medicine, accessed 1 November 2021); https://clinicaltrials.gov/ct2/history/NCT02811 861 ? $\mathrm{A}=20 \& \mathrm{~B}=21 \& \mathrm{C}=$ Side-by-Side\#StudyPageTop

44. Choueiri, T. K. et al. N. Engl. J. Med. 384, 829-841 (2021).

45. US Food and Drug Administration. FDA approves nivolumab plus cabozantinib for advanced renal cell carcinoma. https://www.fda.gov/drugs/resources-information-approveddrugs/fda-approves-nivolumab-plus-cabozantinib-advancedrenal-cell-carcinoma (2021).

46. Motzer, R. et al. N. Engl. J. Med. 384, 1289-1300 (2021).

47. US Food and Drug Administration. FDA approves lenvatinib plus pembrolizumab for advanced renal cell carcinoma. https://www.fda.gov/drugs/resources-information-approveddrugs/fda-approves-lenvatinib-plus-pembrolizumab-advancedrenal-cell-carcinoma (2021).

48. Strohbehn, G. W. \& Goldstein, D. A. Nat. Rev. Clin. Oncol. 18, 395-396 (2021).

49. National Cancer Institute. Vol. 2021 (2021) 2019 NCI Budget Fact Book: Cancer Centers. https://www.cancer.gov/about-nci/budget/ fact-book/extramural-programs/cancer-centers (2019).

50. US Patent and Trademark Office. Statement by Director Iancu before the United States Senate Subcommittee on Intellectual Property, Committee on the Judiciary. https://www.uspto.gov/ about-us/news-updates/statement-director-iancu-unitedstates-senate-subcommittee-intellectual (2019).

51. A study of pembrolizumab with or without lenvatinib as first line (1L) intervention in a programmed cell death-ligand 1 (PD-L1) selected population with recurrent or metastatic head and neck squamous cell carcinoma (LEAP-010). National Clinical Trials Registry (accessed 1 November 2021); https://clinicaltrials.gov/ ct2/show/NCT04199104

52. Efficacy and safety of pembrolizumab with lenvatinib vs. docetaxel in participants with metastatic non-small cell lung cancer (NSCLC) and progressive disease after platinum doublet chemotherapy and immunotherapy (LEAP-008). National Clinical Trials Registry (accessed 1 November 2021); https://clinicaltrials. gov/ct2/show/NCT03976375

53. Efficacy and safety study of pembrolizumab with or without lenvatinib in adults with programmed cell death-ligand 1 (PD-L1)-positive treatment-naive non-small cell lung cancer (LEAP-007). National Clinical Trials Registry (accessed 1 November 2021); https://clinicaltrials.gov/ct2/show/NCT03829332

54. Safety and efficacy of lenvatinib in combination with pembrolizumab versus lenvatinib as first-line therapy in participants with advanced hepatocellular carcinoma (LEAP-002). National Clinical Trials Registry (accessed 1 November 2021); https://clinicaltrials.gov/ct2/show/NCT03713593

55. Safety and efficacy study of pembrolizumab combined with lenvatinib as first-line intervention in adults with advance melanoma (LEAP-003). National Clinical Trials Registry (accessed 1 November 2021); https://clinicaltrials.gov/ct2/show/NCT03820986

56. Safety and efficacy study of pemetrexed + platinum chemotherapy + pembrolizumab with or without lenvatinib as first-line intervention in adults with metastatic nonsquamous non-small cell lung cancer (LEAP-006). National Clinical Trials Registry (accessed 1 November 2021); https://clinicaltrials.gov/ct2/show/NCT03829319

57. Study of first-line pembrolizumab with lenvatinib in urothelial carcinoma cisplatin-ineligible participants whose tumors express programmed cell death-ligand 1 and in participants ineligible for platinum-containing chemotherapy (LEAP-011). National Clinical Trials Registry (accessed 1 November 2021); https://clinicaltrials.gov/ct2/show/NCT03898180 
58. Lenvatinib/everolimus or lenvatinib/pembrolizumab versus sunitinib alone as treatment of advanced renal cell carcinoma (CLEAR). National Clinical Trials Registry (accessed 1 November 2021); https://clinicaltrials.gov/ct2/show/NCT02811861

59. Pembrolizumab and lenvatinib in participants with hepatocellular carcinoma (HCC) before liver transplant (PLENTY202001). National Clinical Trials Registry (accessed 1 November 2021); https://clinicaltrials.gov/ct2/show/NCT04425226

60. Lenvatinib in combination with pembrolizumab versus treatment of physician's choice in participants with advanced endometrial cancer (KEYNOTE-775). National Clinical Trials Registry (accessed 1 November 2021); https://clinicaltrials.gov/ct2/show/ NCT03517449

61. Pembrolizumab plus lenvatinib versus chemotherapy for endometrial carcinoma (LEAP-001). National Clinical Trials Registry (accessed 1 November 2021); https://clinicaltrials.gov/ ct2/show/NCT03884101

62. Safety and efficacy of lenvatinib with pembrolizumab in combination with transarterial chemoembolization in participants with incurable/non-metastatic hepatocellular carcinoma (LEAP-012). National Clinical Trials Registry (accessed 1 November 2021); https://clinicaltrials.gov/ct2/show/ NCT04246177

63. FDA. FDA approves avelumab plus axitinib for renal cell carcinoma. (15 May 2019); https://www.fda.gov/drugs/resourcesinformation-approved-drugs/fda-approves-avelumab-plusaxitinib-renal-cell-carcinoma

64. Garon, E. B. et al. Lancet 384, 665-673 (2014)

65. Peer, C. J. et al. Br. J. Clin. Pharmacol. 86, 1769-1777 (2020).

66. A study to compare treatments for a type of kidney cancer called TFE/translocation renal cell carcinoma (tRCC). National Clinical Trials Registry (accessed 1 November 2021); https://clinicaltrials. gov/ct2/show/NCT03595124

Acknowledgements

The authors wish to thank L. Yang for assistance with patent research, as well as N. Brown and M. Dorji for assistance with legal research. G.W.S. is an employee of the US Federal Government; the views expressed in this manuscript do not reflect the views of the US Federal Government and are his personal views.

Author contributions

All authors meet International Committee of

Medical Journal Editors criteria for authorship in the presented work.

Competing interests

The authors declare no competing interests.

Additional information

Supplementary information The online version contains supplementary material available at https://doi.org/ 10.1038/s41587-021-01137-6. 